\title{
El huerto escolar: estrategia didáctica para la promoción de valores ambientales en la educación inicial
}

\author{
The school garden: didactic strategy for the promotion of \\ environmental values in initial education
}

\author{
Blanca Acuña \\ 1973bk33@gmail.com \\ Código ORCID: 0000-0002-8466-1898 \\ Universidad Pedagógica \\ Experimental Libertador, Venezuela
}

\author{
Cristian Sánchez \\ csanchezipc@gmail.com \\ Código ORCID: 0000-0002-7471-0107 \\ Universidad Pedagógica \\ Experimental Libertador, Venezuela
}

Artículo recibido en abril 2019 | Arbitrado en mayo 2019 | Publicado en julio 2019

\section{RESUMEN}

Los docentes deben comprometerse con la educación ambiental, propiciando el contacto de sus alumnos con la realidad socionatural escolar y su participación en la toma de decisiones para la solución de problemas ambientales de su entorno. El propósito de la investigación, realizada en el Jardín de Infancia Dr. José Gregorio Hernández, Los Teques (Miranda, Venezuela), fue desarrollar actitudes y valores ambientales a través del huerto escolar como estrategia de aprendizaje en la educación ambiental no formal. La investigación fue de carácter cualitativo, de campo, con diseño de investigación-acción participativa, fundamentada en la teoría constructivista y en el paradigma fenomenológico. La participación incluyó a los investigadores, alumnos de Educación Inicial y sus representantes. El estudio se desarrolló en cinco etapas. La efectividad del huerto escolar se demostró con el análisis de registros fotográficos, diagnósticos pedagógicos y procesos de categorización. Se elaboró un modelo para describir la importancia de esta estrategia didáctica.

Palabras clave: actitudes y valores ambientales; educación ambiental no formal; educación inicial: huerto escolar: Venezuela

\section{ABSTRACT}

Teachers must commit to environmental education, promoting the contact of their students with the school social reality and their participation in decision-making for the solution of environmental problems in their environment. The purpose of the research, developed in the Dr. José Gregorio Hernández Kindergarten, Los Teques (Miranda, Venezuela), was to develop environmental attitudes and values through the school garden as a learning strategy in non-formal environmental education. The research was of a qualitative, field nature, with participatory action research design, based on constructivist theory and the phenomenological paradigm. Participation included researchers, Early Education students and their representatives. The study was developed in five stages. The effectiveness of the school garden was demonstrated with the analysis of photographic records, pedagogical diagnoses and categorization processes. A model was developed to describe the importance of this didactic strategy.

Keywords: environmental attitudes and values; non-formal environmental education; initial education; school garden; Venezuela 


\section{INTRODUCCIÓN}

Más allá de la simple adquisición de conocimientos y de la transmisión de contenidos académicos dentro del ámbito formal escolarizado, la educación debe propiciar el desarrollo de valores ambientales sustentables, para lo cual el huerto escolar puede constituir una estrategia didáctica ambiental apropiada a los fines de desarrollar un plan educativo que tenga sustentabilidad en el tiempo.

La acción educativa en materia ambiental debe contribuir al desarrollo de conocimientos, actitudes y competencias necesarias para la conservación y mejora del ambiente, además de promover en los estudiantes metodologías que permitan una visión de los problemas ambientales y sus posibles soluciones, de acuerdo al nivel de desarrollo del educando, tanto en el ámbito formal como en el no formal (Carrero y García, 2008).

Se espera que las instituciones educativas desarrollen un tipo de educación en el ámbito no formal, en el que participen todos los integrantes de la comunidad escolar y que cultive nuevas formas de conocimiento y relaciones sociales para la formación de un ser humano capaz de desarrollar prácticas, habilidades, destrezas, cambios de comportamientos y formación de valores dirigidas al fomento de nuevas formas de relación con el ambiente, desde sus primeros años y sus particulares competencias (Luna, 2011).

Siguiendo a Barraza (1998), las escuelas son determinantes en la enseñanza de valores ambientales. Si sus proyectos $\mathrm{y}$ planificaciones educativas y todo el contexto escolar se desarrolla bajo un sistema educativo ambiental, la influencia que ejercerá sobre los niños y niñas será potencialmente positiva para con el ambiente y su proceso social, pues el niño almacena, recupera y activamente manipula la información que se incrementará con la edad, es por ello que resulta efectivo desarrollar valores ambientales en los primeros años de vida.

De allí que el propósito de la investigación se centró en el huerto escolar como estrategia didáctica para evidenciar la formación de actitudes y valores ambientales en los niños y niñas de Educación Inicial, así como rescatar y mantener espacios y áreas verdes de la escuela y crear conciencia ambiental en los estudiantes de Educación Inicial y en el resto de la comunidad escolar.

Todos los cambios y avances sociales, bien sea políticos, económicos, ambientales, culturales, entre otros, inician con la creación y promoción de nuevos conceptos, ideas y proyectos relacionados con la educación, esto con el fin que las instituciones escolares no 
estén ausentes de la realidad ambiental cambiante en su contexto educativo. Se hace necesario, entonces, asumir un concepto de ambiente en concordancia con el enfoque de Educación Ambiental que orienta esta investigación (Left, 2002).

Dentro de ese concepto de Educación Ambiental, este trabajo se orientó bajo la tendencia de la Educación Ambiental para la Sustentabilidad (EDS) (Left, 2002; Zabala y García, 2008), el cual plantea que la (EDS) está encaminada hacia la búsqueda de mejorar la capacidad de análisis, dando un nuevo sentido al pensamiento crítico, conductual $y$ reflexivo del estudiante. Se orienta hacia la búsqueda de nuevas prácticas pedagógicas, donde exista una relación entre la producción de conocimiento y la transmisión y diseminación del saber ambiental, incorporando valores ambientales y nuevos paradigmas de conocimientos en cuanto a la formación educativa ambiental y el desarrollo sustentable. Lo que implica la ejecución de proyectos nacionales, regionales, locales y escolares por parte del Estado y las comunidades, donde la Educación Ambiental sea definida bajo los criterios de la sustentabilidad, y así propicie la conciencia ecológica, valores culturales correspondientes de cada región y una Educación Ambiental que desarrolle conciencia y capacidad para apropiarse de su ambiente.
Por consiguiente, se espera que las instituciones educativas también fomenten un tipo de educación en el ámbito no formal, donde participen todos los integrantes de la comunidad escolar que cultiven nuevas formas de conocimiento y relaciones sociales para la formación de un ser humano capaz de desarrollar prácticas habilidades, destrezas, cambios de comportamientos y formación de valores dirigidas al fomento de nuevas formas de relación con el ambiente, desde sus primeros años y sus particulares competencias (Luna, 2011).

Las escuelas son determinantes en la enseñanza de valores ambientales. Si sus proyectos y planificaciones educativas $\mathrm{y}$ todo el contexto escolar se desarrolla bajo un sistema educativo ambiental, la influencia que ejercerá sobre los niños y niñas será potencialmente positiva para con el ambiente y su desarrollo social, pues, el niño procesa, almacena, recupera y activamente manipula la información que se incrementará con la edad, es por ello que resulta efectivo desarrollar valores ambientales en los primeros años de vida (Barraza, 1998).

En este orden de ideas, Bellorín (1995) expresa que la educación ambiental propende a formar un educador con novedosas concepciones sobre los sistemas ecológicos, que incorpore $y$ resguarde los valores morales y la 
importancia de cada especie que puebla la Tierra.

Cabe mencionar que en Venezuela se han realizado esfuerzos para promover e incorporar la formación ambiental dentro del proceso educativo desde la Educación Preescolar o más recientemente Educación Inicial, hasta el nivel universitario. Con este propósito, Guevara (2004) menciona como logro la inclusión definitiva de la Educación Ambiental en los planes y programas de la Educación Inicial y Básica, los cuales entraron en vigencia en el período 198687, cuando se estableció el compromiso de los organismos del Estado para garantizar la incorporación de la Educación Ambiental en el diseño y administración del currículo de los diferentes niveles y modalidades del Sistema Educativo.

En ese mismo contexto, la Guía Práctica de Actividades para niños de Preescolares (Ministerio de Educación, 1989) plantea "fomentar el desarrollo de actividades e intereses positivos en el niño hacia los valores de su lengua, de su cultura y de su medio ambiente" (p.17), además de "fomentar actitudes que favorezcan la conservación, defensa y mejoramiento del ambiente, así como el uso racional de los recursos naturales" (p.18).

Objetivos que se deben implementar tomando en cuenta las necesidades e intereses de los niños en función de su desarrollo psicoevolutivo y del ambiente donde se desenvuelva. En este contexto la dimensión ambiental debe desarrollarse dentro y fuera del ámbito escolar. Para ese mismo año de 1989, el Currículo de Educación Preescolar (Ministerio de Educación, 1989) expresa, entre sus objetivos generales, la importancia de "fomentar actitudes que favorezcan la conservación, defensa y mejoramiento del ambiente, así como el uso racional de los recursos naturales" (p.125).

Al respecto (Barraza, ob. cit.) manifiesta que deben existir programas de Educación Ambiental donde se involucren los padres de manera consciente y responsable. "Las escuelas como instituciones sociales deben evaluar sus programas y dirigir su atención al desarrollo paralelo de la formación de valores y a la educación ambiental” (p. 22).

Por otra parte, en el Currículo de Educación Inicial (Ministerio de Educación y Deportes, 2001), se indica que "el ambiente es considerado como un todo, con una connotación ecológica. Lo que implica la oportunidad de colocar al niño frente a sus experiencias de aprendizaje con el medio físico, social, y natural que lo rodea” (pp. 56-57).

Para el año 2012, el Gobierno Bolivariano de Venezuela pone en marcha la Guía Pedagógica-Didáctica 
Etapa Preescolar, que conceptualiza al ambiente como un todo, indicando que los niños deben seguir construyendo sus propios aprendizajes bajo experiencias significativas desarrolladas por el docente dentro y fuera del aula (MPPE, 2012).

La Educación Inicial, como su nombre lo indica, es fundamental en el proceso educativo del niño, sus primeras experiencias establecen los cimientos en la vida futura, por lo tanto la Educación Ambiental debe comenzar desde el nivel inicial. Es por eso que la Educación Ambiental en Venezuela, especialmente, la del nivel inicial, debería desarrollarse en un contexto dinámico, cambiante, basado en la realidad de los niños y buscando enriquecer su desarrollo cognitivo, sus habilidades, actitudes $y$ valores (Boada y Escalona, 2003; Pereira, 1997).

Por otra parte, Ruíz (2004) asevera que la Educación Ambiental, no se circunscribe a una asignatura, y debe ser globalizadora multidisciplinaria $y$ universal, ya que no debe verse como algo separado o aislado de la educación.

Al respecto la Ley Orgánica del Ambiente (2006) en el artículo 35, manifiesta que "se debe incorporar en el pensum educativo una asignatura en materia ambiental en todos los niveles y modalidades del sistema educativo, con el fin de formar ciudadanos ambientalmente responsables" (s.n), de igual manera señala que se deben desarrollar procesos ambientales educativos en el ámbito no formal buscando la participación ciudadana.

Por su parte, el artículo 107 de la. Constitución de la República Bolivariana de Venezuela (2009), puntualiza que la Educación Ambiental debe impartirse de manera obligatoria, desde Educación Inicial hasta la universidad, así como también desde el ámbito no formal. Mientras que la Ley Orgánica de Educación (2009), contempla en el artículo 15, entre los fines de la educación que se "debe impulsar la conciencia ecológica con la intención de preservar las especies y el uso racional de los recursos naturales" (s.n).

Una de las estrategias para incorporar de manera práctica en el sistema escolar venezolano, la formación de valores, actitudes y prácticas amigables con el ambiente, con sentido productivo $y$ sustentable inicia en el año 2009 cuando el Ministerio del Poder Popular para la Educación (MPPE), lanza el Programa Todas las Manos a la Siembra (PTMS, 2009), en todo el sistema educativo venezolano, ratificando dichos programas mediante las resoluciones 024(MPPE, 2009) y la Circular 001(MPPE, 2016), donde se pone de manifiesto que las instituciones educativas deben "abrirse camino con otros enfoques, lógicas y métodos que 
permitan formar produciendo y producir formando nuevas lógicas curriculares que le devuelvan al ser humano su capacidad de producir, crear, dar, compartir, convivir"(s.n) y, de esta manera, este programa

plantea incidir en la cultura
escolar generando procesos
de investigación, la siembra
como práctica cotidiana y
generadora de aprendizajes
pertinentes y ajustados a
todas las áreas de formación,
por lo tanto no debe verse
como separado de lo
curricular y la cultura escolar
y comunitaria en general
(s.n).

Siendo en la práctica una forma diferente de Educación Ambiental dentro de un enfoque sustentable (MPPE, 2016). Todo lo anterior sustenta el huerto escolar como una de las estrategias didácticas más expedita para hacer cumplir los lineamientos del PTMS.

Algunas experiencias significativas sobre huertos escolares han sido realizadas por diversos autores, así Hernández (1995) realizó un Plan de Acción, donde el huerto es utilizado como estrategia de integración escuela y comunidad en beneficio de la educación y formación integral de los estudiantes, a través de la participación activa de los docentes, alumnos, padres y representantes.
A su vez, Jiménez (2000) desarrolló una guía didáctica sobre el huerto escolar en Educación Infantil, para que el docente utilice el huerto no solo para la obtención de plantas, sino con el objeto de desarrollar en los niños y niñas amor al trabajo, interés por la investigación, el respeto, actitudes y valores positivos hacia el entorno, hacia sí mismo y los demás.

El huerto escolar como espacio para el aprendizaje de valores ambientales históricamente tuvo otro propósito. Al respecto la Organización para las Naciones Unidas para la Agricultura y la Alimentación (FAO, 2004), menciona que los huertos escolares estuvieron destinados para propiciar conocimientos de biología, matemáticas, recursos naturales, química y otros, es decir eran concebidos como laboratorios, o para producir alimentos con fines demostrativos de las prácticas agrícolas tradicionales.

Otro aporte importante para esta investigación fue La Propuesta de Educación Ambiental en Centros Escolares, conocida como Plan Columela, desarrollada en la provincia de Granada, en Andalucía (España), donde se expresa que si bien, en el huerto escolar se producen experiencias científicas, también se genera un proceso de reflexión, conciencia, y promoción de un pensamiento crítico y un 
compromiso ante la vida cotidiana (Grupo de Cooperación Columela, 2006).

También es oportuno mencionar la experiencia de Hernández (2007), en el estado Zulia, promoviendo la enseñanza ambiental a través del huerto escolar en Educación Básica, con estrategias de aprendizajes para familiarizar a los estudiantes con los principios de la agricultura $y$, el diseño del huerto escolar para el aprendizaje ambiental en la escuela.

De igual manera, García (2009), en Puerto Rico, planificó un huerto escolar como herramienta didáctica para desarrollar la Educación Ambiental, involucrando a la comunidad escolar en la construcción del huerto y en el diseño de una guía sobre el uso de dicho espacio.

Por su parte la Organización para las Naciones Unidas para la Agricultura y la Alimentación-FAO (2009), propuso en Santo Domingo una guía sobre el huerto escolar como recurso de enseñanza y aprendizaje de las asignaturas del Currículo de Educación Básica; dirigida a los docentes, cuyos objetivos fundamentales incluían aprender sobre huertos escolares, educación, actitudes positivas, esparcimiento, diversión $y$ juego, conciencia medio ambiental, niños sanos, beneficios para la comunidad y la escuela, y comercio e ingresos escolares.
Por su parte, Duarte (2011) realizó una investigación, donde el huerto escolar es aprovechado como alternativa para preservar el medio ambiente en la comunidad escolar donde los estudiantes aprendieron a manipular herramientas y materiales requeridos para el huerto, desarrollando actividades de aprendizaje y recreación y como centro de interés, reflexión, amor y comprensión hacia la naturaleza, donde los padres se involucraron de manera satisfactoria en las actividades.

Otra experiencia venezolana reciente y exitosa, que evidencia la importancia del huerto escolar para la Educación Ambiental Comunitaria y para la sustentabilidad del Programa de Alimentación Escolar Bolivariana (PAEB) en San Isidro de Galipán (estado Vargas) fue la realizada por Marín (2015), donde con la participación de la comunidad educativa se logró el cultivo de 26 rubros agrícolas, un recetario y un calendario agrícola para Galipán, además de un modelo teórico educativo ambiental.

De aquí que se consideró altamente recomendable la construcción de un espacio en las instituciones educativas, para el desarrollo de huertos escolares, donde los alumnos, sus padres $y$ docentes realicen la siembra de plantas de ciclos cortos, de importancia económica y alimentaria, aprender sobre la importancia de las plantas para 
mejorar la calidad de vida, valorarlas, además de contribuir al rescate de las áreas verdes en la escuela y la comunidad.

Con base en lo antes expuesto, el propósito de la investigación apuntó a utilizar los espacios abiertos y zonas verdes del Jardín de Infancia Dr. José Gregorio Hernández, ubicado en Los Teques, estado Miranda (Venezuela), para la construcción de un huerto escolar que cumpliera con los propósitos expresados.

Las circunstancias más notoria que justifican la investigación en el Jardín de Infancia Dr. José Gregorio Hernández, incluyen la presencia de espacios abiertos y zonas verdes en mal estado y abandonados; no aprovechados de manera adecuada para actividades que despierten en los estudiantes la motivación por el cuidado del ambiente, aunado a que los docentes no muestran mayor interés en planificarlas, dando como resultado la proyección de un ambiente poco agradable hacia su entorno inmediato; falta de motivación y dedicación para el trabajo educativo ambiental en la institución; poco cuidado y mantenimiento de las áreas verdes por parte del personal de la institución, representantes y estudiantes, así como desinterés en los docentes para la ejecución de actividades educativas ambientales.
Por los hechos anteriormente planteados, se hizo necesario el diseño y construcción de un huerto escolar siguiendo los lineamientos del Programa Todas las Manos a la Siembra (PTMS), con el objeto de formar actitudes $y$ valores ambientales en los niños y niñas de Educación Inicial, además de rescatar y mantener espacios y áreas verdes de la escuela, y sobre todo crear conciencia ambiental primeramente en los estudiantes de Educación Inicial y posteriormente en el resto de la comunidad escolar.

Sobre las bases anteriores se formularon las siguientes interrogantes:

¿Cuáles son las características socionaturales y socio educativas del Jardín de Infancia Dr. José Gregorio Hernández, estado Miranda, que justificarían la construcción y manejo de un huerto escolar como estrategia didáctica para la formación de actitudes y valores ambientales en el ámbito de la Educación Ambiental no Formal?

¿Cuál podría ser la estructura del huerto escolar, construido y manejado con la participación de los miembros de la comunidad escolar: docentes, estudiantes y representantes de Educación Inicial, utilizando plantas ornamentales, medicinales, frutales $y$ de valor alimentario? 
¿Cómo se evidenciaría la formación de valores y actitudes ambientales en los niños de Educación Inicial que participaron en el desarrollo del Huerto Escolar?

\section{MÉTODO}

Por cuanto el propósito de la investigación fue construir un huerto escolar como estrategia didáctica de la Educación Ambiental no formal, dirigido a la formación de actitudes y valores ambientales en Educación Inicial, se realizó una investigación de campo, conforme a su definición en UPEL, 2012.

El nivel de la investigación de campo, de carácter cualitativo, fue el proyectivo, pues permitió estructurar un huerto escolar para facilitar aprendizajes que permitieron evidenciar la formación de valores y conocimientos ambientales y de respeto hacia el ambiente, además de contribuir estéticamente a mejorar la calidad de vida de los miembros de la comunidad escolar.

El diseño de la investigación fue no experimental, centrada en la investigación acción participante, en la cual, con la participación del investigador se indagó sobre "situaciones sociales y educativas percibidas como problemáticas, susceptibles de cambios y necesitadas de alternativas operativas" (Boggino y Rosekrans, 2004, p.50); se orienta hacia la resolución de problemas mediante un proceso cíclico que va desde la actividad reflexiva a la actividad transformadora (Rodríguez y Valldeoriola, 1999).

A propósito, autores como Salgado (2007), sostienen que la investigaciónacción participante permite además resolver problemas cotidianos $\mathrm{e}$ inmediatos, a la vez que mejorar prácticas concretas, con la permanente participación de los actores sociales en el proceso de investigación, considerándolos actores inmediatos del proceso y de los resultados.

La investigación se realizó en el Jardín de Infancia Dr. José Gregorio Hernández, ubicada en el barrio José Gregorio Hernández, vía San Pedro de los Altos, municipio Guaicaipuro, parroquia San Pedro, Los Teques, estado Miranda (Venezuela), A su alrededor se encuentran otras comunidades como Aquiles Nazoa, Colinas del Paso, Santa Eduvigis, Colinas del Ángel, residencias Río Arriba y el conjunto Residencial Solar de la Quinta. En las cercanías se encuentran otras instituciones educativas, entre las que se pueden mencionar: Escuela Juan de Dios Guanche, CEI Los Teques, CEI Río Arriba, Escuela Estadal Colina del Ángel, E.B. Dr. José Gregorio Hernández, todas pertenecientes al circuito escolar $\mathrm{N}^{0}$ 17. También se encuentra muy cerca de la comunidad la estación de 
bomberos de Los Teques, la Universidad Simón Rodríguez, así como algunas industrias y comercios.

La Escuela Básica Dr. José Gregorio Hernández fue fundada en el año 1968, con algunas situaciones irregulares de infraestructura $y$ de espacio, sin embargo la institución ha logrado consolidarse con el paso de los años. En la actualidad el plantel cuenta con 14 docentes, un director y una matrícula de cerca 300 niños y niñas en educación básica, en dos turnos.

El grupo participante estuvo conformado por los investigadores, miembros voluntarios de la comunidad escolar y los alumnos de Educación Inicial que participaron de las distintas actividades para la construcción y manejo didáctico del huerto escolar.

Las técnicas de investigación seleccionadas fueron: la observación, grupos focales de discusión, visitas de campo, matrices FODA, talleres teóricoprácticos y la técnica de análisis de contenidos. Se aplicaron instrumentos como matrices de registro de FODA, registros fotográficos, registros diarios y anecdóticos e informes pedagógicos de los estudiantes de Educación Inicial.

La estrategia didáctica aplicada fue el huerto escolar. Se define estrategia didáctica como el conjunto de acciones planificadas por el docente con el objetivo de que el estudiante logre la construcción del aprendizaje y se alcancen los objetivos planteados. Una estrategia didáctica es, en un sentido estricto, un procedimiento organizado, formalizado y orientado a la obtención de una meta claramente establecida. Su aplicación en la práctica diaria requiere del perfeccionamiento de procedimientos y de técnicas cuya elección detallada y diseño son responsabilidad del docente (UNED, 2013, pp. 1-2).

La investigación se realizó en seis etapas, las cuales se subdividen en fases, y éstas a su vez en actividades, siguiendo el criterio de Marín (2015), con las modificaciones pertinentes.

\section{Diagnóstico de la realidad socio natural y educativa de la institución}

\subsection{Fase de revisión documental}

Esta fase se ejecutó a lo largo de todo el proceso de diseño y construcción del huerto escolar con el fin de apoyar documentalmente todo lo concerniente a la parte histórica, ambiental, teoría, aspectos legales y metodológicos, así como también evidencias de carácter documental y fotográfico que le aporten sustentos de aspecto referencial en el momento de analizar los resultados de la investigación. La revisión documental abordó antecedentes localización geográfica de la institución, evidencias 
fotográficas de la realidad socionatural del colegio, reseña histórica de la institución.

\subsection{Fase de diagnóstico de la realidad socionatural y socioeducativa}

\subsubsection{Acercamiento a la Institución}

\section{Educativa}

Se realizaron reuniones con el personal directivo de la institución para informarles sobre el proyecto, sobre los siguientes aspectos: a) Explicación del objetivo fundamental de la investigación; b) Relevancia e importancia de lo que se desea lograr a través del huerto escolar; c) Cronograma de actividades a ejecutar. d) Solicitud de apoyo para la realización de las diversas actividades; e) Invitación a participar activamente en el proyecto.

\subsubsection{Reconocimiento del entorno socionatural y socioeducativo de la institución}

Se ejecutaron actividades de campo para reconocer la realidad socionatural $y$ socioeducativa de la escuela con el fin de conocer las condiciones actuales de los espacios y áreas verdes y desocupadas de la institución, mediante fotografías para recoger evidencias graficas que pudieran servir de recursos para la creación del huerto escolar. $\mathrm{Al}$ respecto se realizaron actividades de reconocimiento, como la determinación de las características actuales del colegio: ubicación geográfica de la institución, planta física, aéreas verdes y matricula; disposición de desechos, limpieza, etc.; condiciones socioeducativa: grados escolares $y$ matricula actual de la institución, específicamente del nivel de Educación Inicial.

\section{Sensibilización y motivación}

La finalidad de esta fase de reuniones de trabajo fue brindar información a los miembros de la comunidad escolar acerca de la importancia de un huerto escolar para que a su vez que ellos incentivaran a los niños y niñas para la participación y colaboración voluntaria. Toda esta fase se realizó mediante grupos focales de discusión (Martínez, 2009) para lograr sensibilización, motivación, promoción y la participación activa de los alumnos, padres, representantes y otros miembros de la comunidad escolar.

En dichos grupos focales también se recabó la opinión acerca de la situación ambiental de la institución, conocimientos acerca de las plantas que se siembran en la zona, conocimientos sobre siembra de plantas con valor ornamental, alimenticio y medicinal, sobre el huerto escolar y su posible participación junto a sus representados en la creación y mantenimiento de uno en la institución, el tipo de plantas que pueden estar en el huerto e importancia 
del huerto para mejorar la calidad de vida escolar.

\section{Estudio de fortalezas, oportunidades,} debilidades y amenazas (FODA) para la creación del huerto escolar como estrategia didáctica para la Educación Ambiental

Posteriormente a las reuniones de trabajo se realizó un taller, utilizando la técnica de los grupos focales de discusión (Martínez, 2010), quien plantea que es "focal" porque centra su atención e interés en un tema específico de estudio e investigación que le es propio, por estar cercano a su pensar y sentir. Y es de "discusión" porque realiza su principal trabajo de búsqueda por medio de la interacción discursiva y el contraste de las opiniones de sus miembros. El papel del investigador no será en este caso externo, pues guiará la discusión, fungirá de relator y redactará las conclusiones para la toma de decisiones.

Durante el taller, el grupo focal de discusión indagó las fortalezas (internas), oportunidades (externas), debilidades (internas) y amenazas (externas) (FODA) de la institución que pudieran incidir positiva y negativamente en la creación y permanencia en el tiempo del huerto escolar, utilizando como instrumento una matriz FODA (Cortadelas, 2006).
La actividad se evaluó mediante el análisis colectivo de las matrices FODA, y conforme a ello se establecieron conclusiones para proponer soluciones que potencien las fortalezas, se aprovechen las oportunidades de apoyo extrainstitucionales, se neutralicen las debilidades de la institución y se ataquen las amenazas externas como vandalismo, robo de insumos $\mathrm{y}$ daños al huerto escolar por agentes externos.

\section{Estructuración del huerto escolar}

\subsection{Planificación del huerto escolar}

El grupo focal utilizó las conclusiones de la discusión de las matrices FODA para escoger, de manera consensuada, el espacio más apropiado en el cual se construiría el huerto escolar. Se definió el diseño del huerto, listado preliminar de materiales y herramientas que se necesitarían y el aporte de los representantes, comunidad, e instituciones públicas y privadas.

\subsection{Taller para la motivación de la} Comunidad Educativa

Se realizó un taller con el Grupo Focal acerca de los aspectos relacionados con el huerto escolar: llantas apropiadas, como ornamentales, de valor alimenticio y medicinal, abonos orgánicos, procedimientos para la siembra, etc. 


\subsection{Construcción del huerto escolar}

Para la ejecución de esta fase se utilizaron las directrices del Programa Todas las Manos a la Siembra, ajustadas al propósito de la investigación, en donde participaron las estudiantes de Educación Inicial con sus padres y representantes, los investigadores y otros miembros voluntarios de la comunidad escolar. Las actividades a realizar serían inicialmente las siguientes:

a. Preparación del lugar destinado para el huerto; limpieza del lugar, preparación de los envases para el compost; colocación de la malla.

b. Recolección de los insumos necesarios para la construcción del huerto escolar con el aporte voluntario de los padres, representantes, de los estudiantes, docentes, voluntarios y entes públicos que puedan prestar apoyo a la investigación.

c. Discusión consensuada para la asignación de responsabilidades en conjunto con los padres, madres, representantes, para el mantenimiento del huerto.

d. Selección de implementos a utilizar: pala, manguera, envases, cestas, otros.

e. Selección de las semillas y plantas que serían sembradas en el huerto escolar.

f. Preparación del abono y sustrato

g. Labores de cultivo de semillas adaptados a la zona

h. Trasplante de plantas germinadas. i. Labores de cultivo: riego, fertilización, abono, desmalezamiento, control de plagas con métodos orgánicos.

j. Registro fotográfico tanto del crecimiento y evolución de las plantas como de la conducta de los estudiantes de Educación Inicial.

\subsection{Actividades para propiciar el desarrollo de actitudes y valores ambientales}

En la medida en que se construía el huerto escolar se realizaron actividades de observación, seguimiento, mantenimiento, educativas y lúdicas, que permitieron evaluar el propósito didáctico del huerto para la Educación Ambiental No Formal en Educación Inicial, como es la formación de valores y actitudes ambientales; mediante su registro en los Diagnósticos Pedagógicos de los estudiantes de Educación Inicial.

\section{Efectividad del huerto escolar para los propósitos de la investigación}

La efectividad del huerto escolar se verificó con el Análisis de Contenidos de los Diagnósticos Pedagógicos, mediante la técnica propuesta por Abela (2002) y de los registros fotográficos que permitieron mediante procesos de categorización y reducción de categorías, obtener las categorías emergentes para el reconocimiento progresivo de actitudes y valores en las niñas y niños de Educación Inicial, utilizándose además un primer proceso de triangulación con fuentes teóricas. Para ello se utilizó la metodología propuesta por Martínez (2009, 2010). 
Posteriormente, mediante procesos de teorización, se elaboraron dos constructos teóricos, siguiendo la metodología propuesta por Sánchez (2015), uno para actitudes y otro para valores que condujeron mediante un segundo proceso de teorización y triangulación, la emergencia de actitudes y valores ambientales.

\section{RESULTADOS}

Siguiendo la corriente constructivista, la investigación procuró desarrollar al máximo las capacidades e intereses del estudiante y se logró dado que el aprendizaje estuvo directamente vinculado con la práctica de construcción del huerto escolar, pues el constructivismo parte de que la creación del conocimiento es más bien una experiencia compartida y no individual tal como se evidenció en el trabajo compartido de los niños de Educación Inicial. Esta interacción motiva al desarrollo de nuevos conocimientos, rasgos y características, lo que implica una relación entre el medio y el estudiante, el cual crea y construye activamente su realidad personal (Araya, Alfaro y Andonegui, 2007).

De la investigación emergió un modelo teórico (Figura 1) como aporte para la implementación efectiva de investigaciones similares.

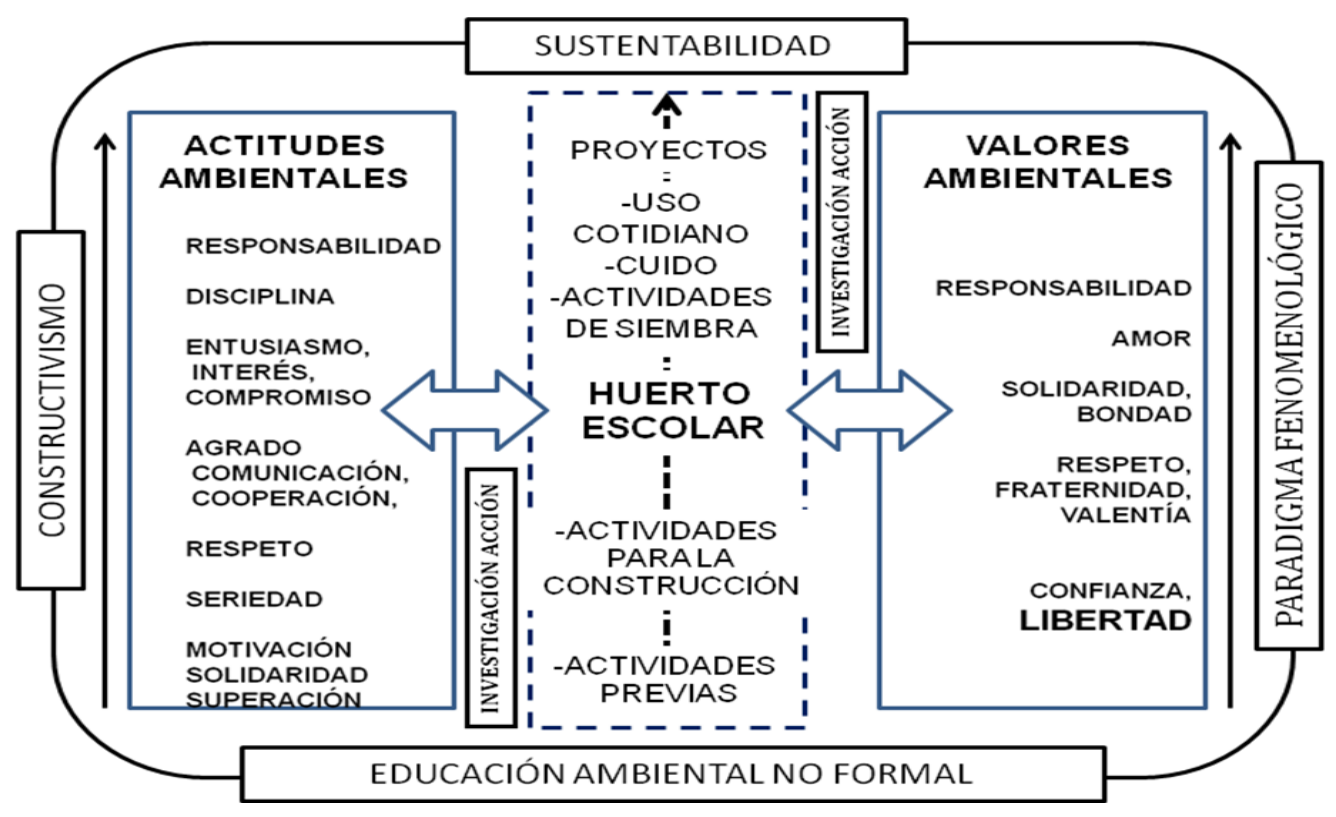

Figura 1. Importancia del huerto escolar para la formación de actitudes y valores ambientales

En la Figura 1 se presenta el modelo teórico que representa la importancia del huerto escolar para la formación de actitudes y valores ambientales, enmarcado en los cuatro grandes soportes de la investigación, vistos de izquierda a derecha y de abajo hacia arriba. 
El constructivismo como teoría educativa porque dicha teoría orientó el "aprender haciendo el huerto de manera progresiva”.

La educación ambiental no formal, cuya dimensión no escolarizada facilitó las actividades fuera del aula.

El paradigma fenomenológico que determinó el comportamiento de los investigadores para dejar fluir libremente los procesos cognitivos $y$ evidenciar sin prejuicios valorativos las actitudes y valores ambientales.

En la parte superior la sustentabilidad como corriente de la educación ambiental, puesto que se aspira que el comportamiento ambiental de los niños así como el producto de su esfuerzo: el huerto escolar, sea sustentable en el tiempo para beneficio de las generaciones futuras de estudiantes de Educación Inicial y de su propio comportamiento ciudadano.

En el recuadro interno de línea interrumpida, de abajo hacia arriba en el sentido de la flecha y teniendo como centro el huerto escolar, se resumen el conjunto de actividades desarrolladas a lo largo del proceso de construcción del huerto.

En sendos recuadros laterales se presentan las actitudes (a la izquierda) y los valores (derecha), que se fueron evidenciando progresivamente durante la construcción y uso cotidiano del huerto escolar.

Las flechas horizontales indican que las actitudes y valores ambientales que no solamente pueden ser el producto de la influencia positiva de la construcción y uso del huerto (sentido hacia afuera de la línea punteada), sino que también se activaron en los niños producto de sus conocimientos previos, experiencias $y$ enseñanzas en su hogar (sentido hacia afuera de la línea punteada) y que permeaban en cada proceso y actividad durante la construcción y uso del huerto escolar.

\section{CONCLUSIONES}

La investigación realizada demostró que la construcción y manejo de un huerto escolar dentro de una institución educativa, puede ser una estrategia didáctica de la educación ambiental no formal, para propiciar el desarrollo de actitudes y valores ambientales, en los estudiantes de Educación Inicial.

Las diversas actividades ejecutadas por los niños del Jardín de Infancia Dr. José Gregorio Hernández, con el apoyo de docentes, padres, representantes $y$ voluntarios, les facilitó la adquisición de aprendizajes cognitivos a la vez que progresivamente mostraron con sus comportamientos, el desarrollo de actitudes $\mathrm{y}$ valores ambientales los 
cuales se esperan sean permanentes en el tiempo, comprobación que requerirá ulteriores investigaciones.

Las actitudes ambientales evidenciadas a lo largo del proceso de construcción y uso cotidiano del Huerto Escolar fueron, en orden de importancia: la responsabilidad, disciplina, entusiasmo, interés, agrado, compromiso, comunicación, cooperación, respeto, seriedad, motivación, solidaridad y superación.

De igual manera, los valores ambientales evidenciados a lo largo del proceso de construcción y manejo cotidiano del huerto escolar fueron: responsabilidad, amor, solidaridad, bondad, respeto, fraternidad, valentía, confianza y libertad.

Es de resaltar que la responsabilidad y el respeto se mostraron igualmente como actitudes y valores, lo que permitió reflexionar acerca que, si se sigue estimulando en los niños la responsabilidad ambiental y el respeto al ambiente en sus instituciones educativas, es posible tener a futuro verdaderos ciudadanos ambientales.

Es imprescindible reconocer que el proceso educativo ambiental evidenciado en los niños de Educación Inicial, estuvo encaminado a procurar que estos desarrollaran progresivamente, mediante las actividades vivenciadas en el huerto escolar, un gran interés por el ambiente, participación activa para cuidar su entorno e interés por la calidad de vida del ambiente escolar.

Todo lo anterior conduce a sostener que la educación ambiental debe ser permanente y progresiva, dirigida no solo a la adquisición de conocimientos, sino también a la formación $y$ consolidación de valores y actitudes ambientales en los niños, e indirectamente a sus padres $y$ representantes, así como a sus maestros $\mathrm{y}$ voluntarios que los acompañen a lo largo de este proceso (Ruiz, 2004).

La propuesta de un modelo teórico como aporte final de la presente investigación pretende mostrar la importancia del huerto escolar para la formación de actitudes y valores ambientales en los niños de Educación Inicial, pero puede ser adaptado para aplicar su metodología en otros niveles y modalidades del sistema educativo venezolano.

\section{REFERENCIAS}

Abela, J. A. (2002). Las técnicas de análisis de contenido. Recuperado de http://public.centrodeestudiosandaluc es.es/pdfs/S200103.pdf

Araya, V., Alfaro, M., y Andonegui, M. (2007). Constructivismo: Orígenes y Perspectivas. Laurus, 3(24), 76-92. Recuperado de http://www.redalyc.org /pdf/761/76111485004.pdf 
Barraza, L. (1998). Conservación y medio ambiente para niños menores de 5 años. Especies, 73), 19-23. Recuperado de http://anea.org.mx /docs/Barraza-Natura.pdf

Bellorín, M. (1995). Educación y calidad de vida en América Latina. Carta Ecológica. Caracas, Venezuela: Lagoven - Editorial Arte, 5(74), 21-22

Boada, D. y Escalona, J. (2003). Pedagogía ambiental en Venezuela y Educación Inicial. Educere Artículos Arbitrados, 8(25), 181-186

Boggino, N. y Rosekrans, K. (2004). Investigación-acción: reflexión crítica sobre la práctica educativa. Rosario, Argentina: Homo Sapiens

Carrero, A. y García, M. (2008). Impacto de un programa educativo ambiental aplicado para promover la participación ciudadana en la zona costera del Estado Miranda. Revista de Investigación, 64, 103-133. Recuperado de http:// www.scielo.org. ve/pdf/ri/v32n64/art06.pdf

Constitución de la República Bolivariana de Venezuela. (2009). Gaceta Oficial $\mathrm{N}^{0} 5.908$ Extraordinaria. Febrero 19, 2009. Caracas: Ediciones EDUVEN

Cortadelas, J. (2006). Proceso de planificación estratégica. Cátedra UNESCO de Dirección Universitaria. Barcelona: Universidad Politécnica de Cataluña

Duarte, B. (2011). El huerto escolar como alternativa para preservar el medio ambiente en la Comunidad Escolar del Taller De Educación Laboral Bolivariano "San Cristóbal". Tesis de Maestría. Universidad Pedagógica Experimental Libertador. Venezuela

García, M. (2009). El huerto escolar como herramienta pedagógica en educación ambiental. Tesis de
Maestría, Universidad Metropolitana San Juan Puerto Rico, Recuperado de http://www.suagm.edu/umet/bibliotec a/UMTESIS/Tesis_Ambientales/educa cion_ambiental/2009/MGarciaCancio 021209.pdf

Grupo de Cooperación Columela. (2006).

La Agricultura Ecológica, una alternativa sostenible. Una Propuesta de Educación Ambiental en Centros Escolares. Recuperado de http://www.huertoalegre.com/assets/P ublicaciones/descargas/-AgriculturaEcolgica.pdf

Guevara, P. (2004). Diagnóstico de la Educación Ambiental en Venezuela. Universidad de Carabobo Facultad de Ingeniería. Recuperado de http://servicio.bc.uc.edu.ve/ingenieria/ revista/a7n2/7-2-5.pdf

Hernández, L. (2007). El huerto escolar como Estrategia para la Enseñanza Ambiental en la Escuela Básica María Camargo de Álvarez. Tesis de Maestría, Universidad Metropolitana San Juan Puerto Rico. Recuperado de http://200.35.84.131/portal/bases/marc /texto/9111-07-02094.pdf

Hernández, M. (1995). El huerto escolar como estrategia de integración escuela comunidad. Tesis de Maestría. Universidad Nacional Abierta. Recuperado de http://biblo.una.edu.ve/docu.7/bases/ marc/texto/t35014.pdf

Jiménez, M. (2000). El huerto escolar en educación infantil. Una propuesta práctica de trabajo. Asociación para el Desarrollo Rural de la Campiña y los Alcores de Sevilla. Recuperado de https://ecohuertosescolares.eu/file/88/ download?token=0_JG5rm8

Ley Orgánica de Educación. (República de Venezuela). ( $\mathrm{N}^{\circ}$ 5929). (2009, 
Agosto 15). Recuperado de http://www ipasme.gob.ve/images/ documents/ley_organica_de_educacio n_-15_08_09.pdf

Ley Orgánica del Ambiente. (República de Venezuela). (Gaceta Oficial de la República Bolivariana de Venezuela $N^{o}$ 5.833). (2006, Diciembre 22).

Recuperado de

http://www.iclam.gov.ve/pdf/leyes_ amb/13_ley_org_amb.pdf

Luna, V. (2011). Educación Ambiental no formal: Fundamentos para la elaboración y diseño de un taller de ciencias para niños. Recuperado de http://digeset.ucol.mx/tesis_posgrado/ Pdf/V eronica_Luna_DIaz.pdf

Marín, Y. (2015). Plan de Acción Educativo Ambiental (PAEA) para la Sustentabilidad del Programa de Alimentación Escolar Bolivariano (PAEB) en la Unidad Educativa Nacional Bolivariana San Isidro De Galipán, Estado Vargas. Tesis de Maestría. Universidad Pedagógica Experimental Libertador. Caracas, Venezuela

Martínez, M. (2009). Comportamiento humano, nuevos métodos de investigación. México: Trillas

Martínez, M. (2010). Nuevos paradigmas en la investigación. Caracas: Alfa

Ministerio de Educación y Deportes (2001). Currículo de Educación Inicial. Caracas: Grupo Didáctico

Ministerio de Educación y Deportes (2005). Bases Curriculares de Educación Inicial. Caracas: Venezuela Ministerio de Educación. Dirección de Educación Preescolar (1989). Currículo de Educación Preescolar. Modelo Normativo. Caracas, Venezuela
Ministerio de Educación. Dirección de Educación Preescolar (1989). Guía Práctica de Actividades para Niños Preescolares. Tomo I. Caracas, Suapi Editores

Organización para las Naciones Unidas para la Agricultura y la Alimentación, FAO. (2004). La mejora de la nutrición y educación infantiles mediante programas de horticultura escolar. Recuperado de http:/ftp.fao.org /docrep/fao/008/af080s/af080s00.pdf

Organización para las Naciones Unidas para la Agricultura y la Alimentación, FAO. (2009). El Huerto Escolar como Recurso de EnseñanzaAprendizaje de las asignaturas del Currículo de Educación Básica. Recuperado dehttp://www.fao.org/ag/humannutriti on/21877061e61334701c700e0f53684 791ad06ed

Pereira, M. (1997). Educación en valores. Metodología e innovación educativa. México: Trillas

Rodríguez, D. y Valldeoriola, J. (1999). Metodología de la Investigación. Recuperado de http://zanadoria.com/syllabi/m1019/m at_cast-nodef/PID_00148556-1.pdf

Ruiz, A. (2004). Educación, Medio Ambiente y Didáctica del Entorno. Teoría y práctica. Madrid: Editorial Popular

Sánchez, C. (2015). Red de Acción Docente para la Reducción de Riesgos Socionaturales y Tecnológicos. Tesis de Doctorado. Universidad Pedagógica Experimental Libertador, Caracas 
UNED. (2013) ¿Qué son las estrategias didácticas? Recuperado de httpwww.uned.ac.cracademicaimages ceceddocsEstaticoscontenidos _curso_2013.pdf

UNESCO. (1986). Programa Internacional de Educación Ambiental 1986-1987. Recuperado de http://unesdoc.unesco.org/images/ 0015/001567 /156731sb.pdf
Zabala, I. y García, M. (2008). Historia de la Educación Ambiental desde su discusión y análisis en los congresos internacionales. Revista de Investigación, 63, 201-218. Recuperado de http://www.scielo. org.ve/pdf/ri/v32n63/art11.pdf 